\title{
La muerte de las reinas. Opúsculos y relaciones italianas durante el Renacimiento
}

\author{
The death of the queens. Pamphlets and Italian relationships \\ during the Renaissance
}

\section{María José Bertomeu Masià}

Universitat de València. m.jose.bertomeu@uv.es

Recibido: 23.06.2019. Aceptado: 24.09.2019

Resumen: La difusión de relaciones sobre bodas, entradas, fiestas o exequias durante el siglo XVI respondía a la enorme demanda del público y contribuyó a la fijación en el imaginario colectivo de la memoria de los poderosos, celebrados en la muerte con toda la pompa de los funerales de estado. En este sentido, son conocidísimos los libros de exequias que narraron las fiestas en honor del funeral del emperador Carlos $\mathrm{V}$ a su muerte en 1558, así como los dedicados a otros monarcas, pero son menos conocidos los funerales de tantas mujeres, reinas y consortes, que adquirieron también categoría de celebración de estado y se difundieron mediante opúsculos, pliegos sueltos o relaciones de sucesos que todavía están hoy por estudiar.

Palabras clave: exequias; reinas; memoria; imagen femenina; relaciones.

\begin{abstract}
The dissemination of pamphlets on weddings, entries, celebrations and funerals during the 16th century responded to the immense demand on the part of readers, and contributed to establishing in the collective imagination a vivid memory of powerful and renowned figures whose deaths received all the pomp of a funeral of State. Wellknown are the books that narrated the festivities in honour of the funeral of Emperor Charles V following his death in 1558, as well as those dedicated to other monarchs. However, less well-known are the funerals of countless women, queens and consorts, who were also granted State celebrations upon their deaths, which were narrated in pamphlets and loose sheets, or relations of events that still remain to be studied.
\end{abstract}

Keywords: funerals; queens; memory; female image; pamphlet. 



\section{Opúsculos, relaciones de sucesos y memoria}

El estudio de hojas volantes, avisos y relaciones sobre damas ilustres impresos durante el siglo XVI es fundamental para profundizar en la construcción de la imagen pública de las mujeres con poder. La difusión de textos escritos se extendió a todos los grupos sociales por efecto de la imprenta lo que contribuyó a transmitir estereotipos y a fijar modelos de comportamiento. Esto adquiere particular relevancia cuando hablamos de las grandes figuras femeninas de nuestro pasado: reinas, gobernantes y consortes en el ejercicio del poder, cuyas acciones, relacionadas o no con el ejercicio de este, se celebraron y difundieron mediante relaciones de sucesos y otro tipo de escritos. Los textos que narraban exequias tuvieron un papel propagandístico y contribuyeron a la fijación en el imaginario colectivo de la memoria de los poderosos, celebrados en la muerte con toda la pompa de los funerales de Estado. En este sentido, son conocidísimos los libros de exequias que narraron las fiestas en honor del funeral del emperador Carlos $\mathrm{V}$ a su muerte en $1558^{1}$, pero son menos conocidos los funerales de tantas mujeres, reinas y consortes ${ }^{2}$, que adquirieron también categoría de celebración de Estado.

Las relaciones de sucesos han comenzado a estudiarse desde hace un tiempo, sobre todo en ámbito hispánico, como un género, entre la literatura y la información, precursor del periodismo moderno, por su papel como herramienta fundamental de difusión, propaganda y fijación de

\footnotetext{
* La investigación presentada en este artículo se ha llevado a cabo en el seno del proyecto de investigación del Ministerio de Ciencia, Innovación y Universidades con referencia FFI2017-83252-P MAUSTRIA. Las mujeres en la Casa de Austria (1526-1600). Corpus documental II, dirigido por Júlia Benavent.

${ }^{1}$ Sobre las celebraciones funerarias de la Casa de Austria se ha escrito bastante literatura científica que aborda distintos aspectos, desde la arquitectura efímera (Allo Manero \& Lorente, 2004, entre muchos otros), desde el estudio de la propia ceremonia (Allo Manero, 1993; Varela, 1990); tanto en Europa como en Latinoamérica, desde el punto de vista de la propaganda o la publicación de opúsculos y otros textos al respecto (Solís de los Santos, 2009; Bertomeu, 2014).

${ }^{2}$ Se pueden consultar artículos puntuales sobre los funerales de algunas mujeres como Juana I de Castilla (Domínguez Casas, 2010) o María Luisa de Orleans (Lopezosa, 2011, entre otros) pero sobre los textos que difundieron el evento no se encuentran estudios con facilidad. Sí sobre otros aspectos, sobre todo las bodas (véase Solís de los Santos, 2007, o Bertomeu, 2009, sobre la de María Tudor y Felipe II; Gómez Salvago, 1998; o Solís de los Santos, sobre la de Isabel de Portugal y Felipe II) o las entradas, casi siempre con ocasión de una boda (Benavent, 2011).
} 
la opinión pública de los poderosos ${ }^{3}$. En ámbito italiano los estudios de este tipo de textos todavía requieren de consolidación, tal es así, que no se ha logrado acordar un nombre definitivo para esta tipología textual si no que se vacila entre 'avvisi a stampa' (Bulgarelli, 1967; Ricci, 2009), 'relaciones', 'Flugschriften' (Amft, 2018; Wilhelm, 1996) o más recientemente 'opuscoli e fogli volanti' (Wilhelm, 2019). Y se transita, además, entre definiciones que convertirían los términos en sinónimos o que los diferencian claramente ${ }^{4}$.

En realidad, como sostienen algunos estudiosos (Wilhelm, 1996; Benavent, 2015), fue en Italia donde primero se comenzaron a imprimir este tipo de obras, ya en los mismos tiempos del nacimiento de la imprenta, si bien con algunas características propias, sobre todo porque comenzaron a difundirse en verso, en forma de lamentos u opúsculos populares épicos que narraban episodios bélicos en octava rima, ligados a las llamadas 'Guerre d'Italia' (Ivaldi, 1989). Según ha explicado recientemente Petta (2017: 523), esta forma versificada convivió en Italia hasta los años 30 del siglo con la composición en prosa, ya con la forma de lo que hoy llamamos 'relaciones de sucesos' que a partir de ese momento se consolidaría como la tipología preferida, diversificando los géneros textuales sobre todo bajo el título de 'avvisi' o 'lettere' o 'copia di una lettera', como las más utilizadas, y dejando ya de lado, en su mayor parte, el verso.

A nuestro juicio, estudiosos como Camilla Amft (2018: 9) o Raymund Wilhelm (2019) definen mejor la realidad tipográfica y cultural italiana al buscar un término más amplio y definir este tipo de comunicación como un producto tipográfico de pocos folios en lengua popular de argumento actual, político o social para influenciar la opinión pública, de coste modesto y amplia difusión, originadas por la reorganización de las formas comunicativas que supuso la llegada de la imprenta. Así, guerras, descubrimientos, desastres naturales o fiestas fueron los temas favoritos.

\footnotetext{
${ }^{3}$ El nacimiento de la SIERS, que organiza un congreso cada tres años junto con el proyecto de la Universidad de Santiago de Compostela, BIDISO, cuya web y base de datos, consultables en el link http://www.bidiso.es/Relaciones/, resultan fundamentales para cualquiera que se acerque a la cuestión de las relaciones de sucesos.

${ }^{4}$ Sobre la posible diferencia entre 'avvisi' y 'relaciones' véase Ettinghausen (2013: 14). Sin embargo, otros autores, como Ricci (2009), usan ambos términos como sinónimos.
} 
Raymund Wilhelm (1996) explicó, además, que estos textos pueden clasificarse en base a dos ejes que se encuentran ya en el título: uno, el del contenido, referido al tipo de evento que narra el texto ('entrata', rotta, vittoria...) y otro relativo a su tipo textual en clave metadiscursiva (lettera, avviso...). Definió también algunas características sobre todo centrándose en la fuerte formularidad que contienen, tanto desde el punto de vista textual, del de las tradiciones discursivas, las dinámicas lingüísticas y los motivos que repiten (Wilhelm, 2019).

Como veremos a continuación, en el caso de los textos centrados en mujeres, encontraremos algunas características propias y, si cabe, aún una mayor estructura formular y voluntad de adhesión a una imagen femenina, difundida y fijada también por los tratados de comportamiento, que tuvieron un auge enorme durante el siglo XVI en Italia ${ }^{5}$.

Estudiar las particularidades de estos textos que preservan y celebran la memoria de las mujeres poderosas frente a los textos escritos para los hombres es fundamental para poder abordar la construcción de la imagen femenina, aquella que nos ha llegado y que ha fijado un ideario tendencioso y articulado en torno a los espacios femeninos que ha distorsionado nuestra visión sobre el ejercicio real del poder que muchas mujeres tuvieron en el pasado.

En este artículo estudiaremos algunas de estas relaciones que honraron la vida de las grandes damas del Renacimiento con ocasión de su muerte. En particular, un grupo de relaciones que narró el funeral de María Tudor junto al del emperador Carlos V; así como las relaciones que narraron las exequias de la archiduquesa Eleonora d'Austria, de la gran duquesa de Toscana, Giovanna d'Austria, o de Caterina d'Austria, entre otras.

\section{Opúsculos y relaciones funerarias sobre mujeres}

E1 21 de septiembre de 1558 moría el emperador Carlos V. Los varios funerales celebrados en su nombre en diversas ciudades europeas tuvieron un enorme eco gracias a los libros de exequias oficiales pero también al tipo de opúsculos y relaciones de sucesos que narraban el

\footnotetext{
${ }^{5}$ Sobre la cuestión de los tratados de comportamiento dedicados a las mujeres véanse Baernstein, 2006; Riondato, 1985; Bertomeu, 2018; Bell, 1999; Kelso, 1978; Murphy, 2001; Pucci, 2015, entre otros.
} 
acontecimiento $^{6}$ que satisfacían la demanda del público por conocer los aspectos más relevantes del evento, como era el caso con ocasión de otros eventos y fiestas (bodas, entradas, victorias bélicas...).

Uno de esos opúsculos narraba los funerales celebrados en Milán el 10 de enero de 1559, 3 meses después de los funerales oficiales en Valladolid y Bruselas celebrados en octubre y diciembre de 1558 respectivamente. Entre ambos funerales, el 17 de noviembre de 1558, había fallecido también María Tudor, reina de Inglaterra y en ese momento esposa de Felipe II. En el funeral milanés en honor del Emperador se aprovechó la ocasión para realizar también el funeral oficial por María, por parte de la Casa de Austria. El texto que da cuenta de ese funeral doble es Essequie celebrate con solenne pompa nella chiesa del Domo di Milano per la Cesarea Maestà Carlo Quinto imperatore romano et per la serenissima regina Maria d'Inghilterra. Nelle quali si descrive il catafalco, con tutto l'apparato della chiesa, et insieme si fa mentione dei nomi di quelle persone honorate che a dette essequie furno presenti. De él hemos encontrado dos testimonios, ambos impresos en Milán apenas acabado el acontecimiento, uno sin impresor y otro de la tipografía de Giovanni Battista Da Ponte et fratelli.

El título incluye elementos que son comunes en esta tipología funeraria y que van desde la descripción de la arquitectura efímera realizada para la ocasión, la decoración, los nombres de personajes importantes que acudieron y, según la extensión del texto, era normal incluir también la descripción de vestimentas, joyas y otros elementos que asentaban en el imaginario colectivo la idea de riqueza y poder tanto del personaje honrado como de los asistentes (Bertomeu, 2014).

Sin embargo, en este caso el título es engañoso, pues la referencia al entierro de María ocupa apenas el último párrafo de la obra, tras varias páginas describiendo la grandiosidad de la arquitectura y las decoraciones, los personajes que acudieron y la fiesta dedicada al Emperador.

Il lunedì seguente furono poi celebrate l'essequie dela sereníssima regina Maria d'Inghilterr. Et era nella chiesa il medesimo apparato del catafalco, panni, lumi, solo era mutate le arme et si era posto al loco delle armi imperiale l'arme del regno d'Ighilterra et nel loco dove erano le colonne col Plus Ultra vi era posto l'arma della rosa rossa ch'è l'insegna della Casa di detta Regina.

\footnotetext{
${ }^{6}$ Me remito a los trabajos citados anteriormente, en la nota 2.
} 
Vi venne l'Illustrissimo Duca di Sessa all'hora medesima che venne a quelle del'Imperator Carlo et vi venne accompagnato dai sopra nominati principi signori officili et gentilhuomini, li quali venero nel habito et nel ordine che di sopra si è narrato. Fu cantata la messa con le medesime cerimonie che fu l'altra, fece l'Oratione l'Illustre signor Pietro Antonio Marliano, senatore, la qual finita, et finita poi la messa, fu posto fine ai funerali.(Essequie celebrate..., 1559, f.3v).

Se aprovechó la arquitectura efímera y la decoración utilizadas para el funeral de Carlos V, cuya descripción no era necesario repetir, pero lo que llama la atención es la ausencia total de cualquier tipo de descripción de la reina, ni física, ni moral ni de su vestimenta, elementos que habían llenado las páginas anteriores referidas al Emperador, que son los que veremos que centran las descripciones en otros textos, y de otras damas. Es cierto que la decepción que había supuesto la boda de María y Felipe, celebrada y difundida también en numerosas relaciones (Solís de los Santos, 2007; Bertomeu, 2009) por la esperanza en la vuelta de Inglaterra a la religión católica y en el nacimiento de un hijo que encarnara la unión, que nunca llegó (Pascual, 2017), son probablemente causa de la desgana por parte de la cancillería austriaca en la honra de la reina que transmite el texto, pero aun así es notable.

Si en el estudio de las relaciones de sucesos en general aún estamos en una etapa de recensio, de localización, catalogación y análisis, más lo estamos en el caso de los textos relativos a las mujeres.

No es posible en el espacio de un artículo abarcar una recensio exhaustiva de opúsculos y hojas volantes sobre la muerte y los funerales de mujeres, ni si quiera si nos centramos solo en mujeres con poder, reinas y otras gobernantes, y a la vez elaborar un estudio filológico completo de los mismos en el sentido planteado al inicio, pero sí creemos que es importante esbozar las líneas maestras a partir de las que se llevará a cabo el análisis.

Para iniciar el estudio nos hemos centrado en la archiduquesa Eleonora de Austria, en Giovanna de Austria, gran duquesa de Toscana, en Ana de Austria, reina de España, y en Caterina de Austria, duquesa de Saboya, aunque hemos localizado en el proceso también opúsculos de este tipo relativos a otras mujeres relevantes de la época como Leonor de Toledo, duquesa de Florencia; María de' Medici, Eleonora d'Este o Lucrezia de' Medici, entre otras. 
Hemos localizado en total 15 textos sobre la muerte y funeral de las damas apenas mencionadas: dos sobre Caterina d'Austria, dos sobre Ana de Austria, cuatro sobre Eleonora de Austria, uno sobre Leonor de Toledo, cinco sobre Giovanna d'Austria y uno sobre María Tudor, que ya hemos referido en líneas anteriores. Todos comparten la actualidad de la información que difunden pues fueron impresos muy cerca del acontecimiento que narran, condición sine qua non, como hemos visto, para la inclusión de los textos en la categoría de relaciones de sucesos.

El de María Tudor es el que ya hemos comentado, y la mención al funeral de la reina es apenas una nota añadida al final, sin ningún tipo de pretensión real de narrar el acontecimiento o describir al personaje para fijar su imagen en la memoria, como hemos visto.

Las dos Orazione dedicadas a Caterina d'Austria, duquesa de Saboya, esposa de Karl Emmanuel, fallecida en 1587, comparten formato textual ${ }^{7}$, propio de los opúsculos funerarios, articulados, en torno a dos ejes, el metadiscursivo, a partir de la palabra 'Orazione' con la fórmula "Orazione di + autor", y el del contenido, expresado por la segunda parte del título "per/fatta + nombre de la persona honrada", y que veremos que se repite en textos dedicados a las otras mujeres estudiadas. Así, por ejemplo, en el caso de Leonor de Toledo, duquesa de Florencia, esposa de Cosimo I de' Medici, muerta en 1562 o en el de Giovanna d'Austria, gran duquesa de Toscana, fallecida en 1578.

Estas oraciones comparten la característica de tener un autor conocido $^{8}$ que se expresa ya en el título, y estar escritas en prosa.

- Caccia, Francesco. 1598. Oratione di Francesco Caccia, dottor di leggi, oratore e consigliere di stato del serenissimo duca di Savoia, Carl Emmanuello, Fatta nella morte della serenissima infante, donna Caterina d'Austria, Duchessa di Savoia. Milano, Padolfo Malatesta.

- Corderi, Girolamo. 1599. Orationi di Girolamo Corderi di Mondoui per l'essequie ordinate in Torino alla serenissima infante donna Caterina d'Austria. Torino, Fratelli Cavalleri.

\footnotetext{
${ }^{7}$ No me detendré en las obras dedicadas a Caterina d'Austria pues se ocupó de ellas Luisella Giachino (2013).

${ }^{8}$ La cuestión de la anonimidad o no de las relaciones ha sido muy debatida, véase, por citar solo un par de trabajos recientes, los textos de Infelise (2017) o Pena Sueiro (2017).
} 
- Adriani, Giovambattista. 1578. Orazione di M. Giovambattista Adriani nell'essequie della serenissima Giovanna d'Austria, gran duchessa di Toscana. Firenze, Stamperia de' Giunti.

- Lottini, Giovanni. 1578. Oratione funerale fatta e recitata nell'Annontiata di Firenze a gl'academici Trasformati da $f$. Giouan'Angelo Lottini scultore per consolarne ogn'animo pietoso dell'immatura, e dannosa morte della sereniss. Giouanna d'Austria gran duchessa di Toscana. Fiorenza, appresso Giorgio Marescotti.

- Mirani, Calderino. 1594. Oratione nella norte et secondo funerale della serenissima manda Eleonora arciduchessa d'Austria. Mantova, appresso Francesco Osanna stampator ducale.

- Vettori, Piero, Orazione di M. Piero Vettori nella morte dell'Illusrissima et eccellentissima donna Leonora di Toledo, duchessa di Firenze. Firenze, figli di Lorenzo Torrentino, 1578.

En cuanto a la estructura del texto encontramos bastante homogeneidad, salvo en un caso, el de la Orazione de Francesco Caccia dedicado a Caterina d'Austria, cuya extensión, además, es mayor que la del resto de textos. Todos comienzan con la dedicatoria en la que se declaran las intenciones y se explica que la composición de la obra tiene lugar con ocasión de la muerte, "la mattina istessa che Firenze onorò publicamente, e publicamente pianse la sua morte" dice, por ejemplo, Niccolò Mini, traductor de la oración de Piero Vettori (1563: 1); luego el texto, que comienza con la invocación a los que escuchan pues fueron compuestas para ser leídas en el propio funeral, como en el caso de las canciones, para pasar después a la loa a la dinastía de la que procede la mujer, la de la casa a la que perteneció por matrimonio, el estado... y, finalmente, a la alabanza de las virtudes de la mujer ideal cristiana del Renacimiento encarnada en la mujer honrada: piedad, caridad, domesticidad, virtudes, además, que en estas grandes damas fueron constantes a pesar de las tentaciones que su posición les presentaba.

Otra fórmula que se repite en los títulos y que une también en el título forma textual y anuncio del contenido es la de la 'Canzone in morte', con la variación 'canzone funebre'. En este caso, encontramos cinco textos, todos en verso, todos breves, impresos en $4^{\circ}$ y de solo 4 folios, y todos de autor conocido. 
- Rai, Flaminio. Post. 1580. Canzone di Flaminio Rai. In morte de la serenissima Anna di Austria regina di Hispagna. Praga: Georgio Nigrino.

- Cresci, Piero. 1595. Canzone funebre nella morte della serenissima madama Eleonora arciduchessa d'Austria, et duchessa di Mantova e di Monferrato. Mantua: Francesco Osanna.

- Comanini, Gregorio (s.a.), Canzone in morte della serenissima madama Eleonora, arciduchessa d'Austria et duchessa di Mantova e di Monferrato. Mantua: Francesco Osanna.

- Ginori, Gino. 1578. Canzone in morte della serenissima regina Giovanna d'Austria gran duchessa di Toscana. Firenze: Giorgio Marescotti.

- Borghini, Raffaello. 1578., Canzone in morte della serenissima reina Giovanna d'Austria, gran duchessa di toscana. Firenze: Giorgio Marescotti.

Las obras dedicadas a Giovanna d'Austria comparten título e impresor. Los dedicados a Eleonora de Austria no comparten título, pero sí impresor, de hecho, como los otros dos textos en su honor, fueron impresos por el tipógrafo ducal Francesco Osanna. El primero, el de Pietro Cresci, dedicado al hijo de la archiduquesa, el Duque de Mantua y Monferrato, y el segundo, el de Gregorio Comanini sin dedicatoria ni colofón. Ambos comparten una representación arquetípica que se ajusta a las virtudes ideales que las mujeres debían poseer. Por una parte, Eleonora es descrita en función de su papel respecto de los hombres la familia: "Madre felice e bella d'eccelsi heroi", "sorella de' principi sublimi" y "alma e serena de' cesari progenie" para acabar alabando su "saper viril sotto donnesco manto" (Cresci, 1595); por otra parte, es representada según los rasgos ideales de la mujer viuda renacentista (Bertomeu, 2018) y, así, se alaba su "maestoso aspetto", "pietoso e divoto pensier", "l'animo grato", "dolce stile", "generoso spirito" y el "magnánimo cor" (Cresci, 1595). Y con esos atributos debe ser recordada, según el escritor, la fama perpetua de su nombre. Es curioso también el inicio de la obra que justifica la redacción en verso por la 'heroica materia' del mismo, lo que lo relacionaría directamente con la tesis de Petta (2017) o Wilhelm (2019) que hacían remitir el origen de las relaciones a la poseía épica consolidada con los textos de ocasión escritos ya desde los inicios de la imprenta en Italia (Bardini \& Beer, 1989). 
Por último, encontramos dos textos bajo la fórmula 'Vita di', con la variante 'Vita et morte'. En este caso se trata de textos más extensos y siempre en prosa que tratan de narrar la vida del personaje antes de pasar a la ceremonia funeraria, aunque esta no siempre es descrita y es sustituida por la narración de la forma en que el personaje afrontó la muerte, de forma cristiana. Los dos textos que hemos encontrado están dedicados a la misma mujer, Eleonora, archiduquesa de Austria. El primero, titulado Vita della serenissima Eleonora Arciduchesa d'Austria, duchessa di Mantova e di Monferrato, compuesta por el jesuita Antonio Folcario, impresa en Mantua, por el tipógrafo ducal Francesco Osanna, en 1598.

El segundo texto es uno de los más interesantes desde el punto de vista del análisis de la imagen de las mujeres y revela hasta qué punto estos textos la estilizaban para acercarla al ideal de la época. Si a esto añadimos el hecho de que todos los textos dedicados a Eleonora, como hemos dicho, fueron impresos por el tipógrafo ducal, la oficialidad de los mismos y su función propagandística quedan claras.

Se trata de la Vita et Morte della serenissima Eleonora arciduchessa di Austria et duchessa di Mantova, compuesta por otro jesuita, Antonio Possevino, e impresa también por el tipógrafo ducal, Francesco Osanna, el 6 de octubre de 1594. Es una obra dedicada, como las otras de este tipo, a loar la vida y los hechos del personaje, en este caso de Eleonora, pero esta tipología se repite por ejemplo en el caso de los textos dedicados a la muerte del emperador Carlos V.

Lo interesante no es tanto la imagen de la duquesa sino su estructura pues está compuesta siguiendo las tres edades de la vida de la mujer que fijaban los tratados comportamentales del siglo: la virgen, la esposa y la viuda ${ }^{9}$. Adriana Chemello (1985:114) ya definió estos estados como "una vera e propria grammatica del comportamento applicata ai diversi strati di donne, conforme alle loro diverse funzioni sociali" con el objetivo de controlar, modelar, prescribir, premiar e inspirar el buen comportamiento cristiano de vírgenes, casadas y viudas de todas las condiciones sociales (Bell, 1999: 6).

\footnotetext{
${ }^{9}$ Sobre literatura comportamental dirigida a las mujeres, además de los textos de Pucci (2015) (2018) y Bertomeu citados, se pueden consultar el de Bell (1999), Murphy (2001) o Samson (2015 a y b).
} 
La imagen que de Eleonora se da en el texto se ajusta perfectamente a los dictados normativos: la virgen, la casada y la viuda, por lo que no hay una descripción de la mujer real, solo una de la mujer ideal: virginal, recatada y pía, dedicada al cuidado de la casa y la familia y, durante su viudedad, a la memoria del esposo fallecido (Pucci, 2012; Bertomeu, 2018). Dedicado al Duque de Mantua y Monferrato, hijo de la fallecida, el texto es extenso, pero fue escrito para ser leído con ocasión de las exequias oficiales como el autor explica al inicio. Primero explica el objetivo del texto "per fare in queste essequie di Madama Eleonora di Austria vostra madre, questo debito ufficio, o di commemoratione et di lode, o de consolatione" (Possevino, 1594: 1r) y, luego, "non paura di attenta audienza, conciosiache già si scorge negli occhi il disiderio o di tutti voi ascoltanti" (Ibid: $1 v$ ).

Tras esto, las tres partes en las que se divide tituladas: "Consideratione del primo stato di virginità nel quale Madama Eleonora di Austria et Gonzaga visse"; "Consideratione del secondo stato nella vita di madama Eleonora di Austria Gonzaga, in tempo che visse maritata" y, por último, "Tempo della viduità della serenissima Madama Eleonora di Austria". La descripción de los tres estados es canónica, basta ver la de la viudedad, en la que, recalca la dedicación de la viuda a la memoria de su esposo y el modelo de piedad que encarnó:

Iddio voleva che nel terzo stato, cioè, di viudità, fosse presente specchio a molti, dapoi lasciasse perpetuo essempio alla posterità di ogni virtù, la quale a tale stato si richiede. Et in questo bastarono sette anni, nei quali visse, i quali con se stessa sacrificò totalmente a Dio rendendosi di giorno in giorno più perfetto holocausto alla divina pietà (Possevino, 1594: 43).

Un poco más adelante "Riprese poi più viva cura del picciolo figliuolo primogenito del presente signor Duca, tenendolo in tal modo per lo spacio di sei anni appresso di se" (Possevino, 1594: 44); y, "fra tanto, come più sciolta al dispregio di se medesima, già portava sotto la soprana veste, vestimenti tanto logori, che mostravano l'allegrezza, la quale ella sentiva in gustare in alcuno modo della povertà di Christo" (Ibid, 45).

Para concluir, Possevino (1594: 81) resume las virtudes de Eleonora en vida: 
[...] è facile all'infinita liberalità di Dio, che le sia stato conceduto di meritare il frutto trentesimo delle maritate, il sessantesimo delle vedove e'l centésimo delle vergini. Già la scienza, la prudenza et l'isperienza non le mancavano $[\ldots]$

Aunque pueda parecer que las virtudes propias de la mujer ideal de la época se conjugan en Eleonora con algunas propias de los grandes hombres, referidas a su capacidad de gobierno (Possevino, 1594: 82):

Non le mancarono parimente gratia, autorità et confidenza. Non è, ascoltanti, cosa la quale discrideti più et ponga in diffidenza un ambasciatore et attraversi maggiormente i negoci ch'un minimo cenno di doppiezza et di proprio interesse, che se a questo si aggiunge l'alterezza di core o per ingratiarsi del prencipe a cui è mandato, scopra i segreti del suo signore, e sparga semi di odio, dove dee procurare la pace dubbio non è ch'egli apporta incredibile danno.

Lo cierto es que esas virtudes, confianza, gracia, autoridad y piedad, redundan en que Eleonora sabía cuándo hablar y cuándo callar y la apariencia que debía dar de su estatus, lo que refleja exactamente las aptitudes de la viuda renacentista, que debía dedicarse a la contemplación y al rezo, a cuidar de la casa y los hijos, no expresar demasiado ni sus opiniones ni sus pensamientos y mantener siempre un comportamiento decoroso y prudente, aceptando, y reflejándolo con su vestimenta incluso, su estado y ser un modelo de piedad maternal y perdurable devoción a la memoria del marido (Bertomeu, 2018).

Estas tres tipologías textuales ${ }^{10}$ que hemos repasado son comunes en los textos dedicados a la muerte de hombres ilustres, como en el caso de Carlos V o Felipe II, por quedarnos en el mismo ámbito dinástico, y la voluntad de proyectar una imagen concreta, estilizada y arquetípica, del

\footnotetext{
${ }^{10}$ Además de estas hemos encontrado un libro de exequias oficial en honor del funeral de Ana de Austria: Descrittione de l'edificio, et di tutto l'apparato, con le cerimonie pertinenti a l'essequie de la serenissima d. Anna d'Austria, regina di Spagna. Celebrate ne la chiesa maggior di Milano, a di VI di settembre MDLXXXI. Opera di m. Pellegrinode' Pellegrini, architetto di sua Maestà, et de la fabrica del duomo di Milano. Milano, Paolo Gottardo 1581; un par de obras poéticas: Un Dialogo d'Arno et di Flora nella norte della lor serenissima gran duchessa Giovanna d'Austria, Regina nata, anónimo, impreso en Florencia por Giuliano Marini, 1578; y un libro de rimas con el título Rime nella norte della serenissima Regina Giovanna d'Austria, granduchessa di Toscana, Michele Capri, Florencia, Bartolomeo Semartelli, 1578.
} 
personaje también lo es, lo que cambia es la imagen en sí. En el caso de Carlos V y Felipe II la del gran hombre de estado cristiano, en el caso de las mujeres, la madre y esposa recatada y dedicada a la oración y al cuidado familiar.

\section{Conclusiones}

La investigación sobre los textos de diversa tipología, dentro de la nueva forma de comunicación conocida como relaciones de sucesos, que sirvieron para fijar en el imaginario colectivo la imagen de muchas mujeres ilustres y su papel en la sociedad tiene un interés notable tanto para sacar a la luz textos que hasta ahora no han recibido la atención de los estudiosos y que, por tanto, contribuyen a completar la imagen cultural del siglo XVI; como para profundizar en cómo se ha construido la imagen de las mujeres con poder que ha llegado hasta nuestros días y que ha oscurecido su labor y su papel real en su tiempo.

Hemos querido contribuir a poblar el corpus de textos escritos sobre mujeres centrándonos en aquellos escritos con ocasión de sus muertes, para celebrar sus vidas y fijar su memoria, y, al tiempo, comenzar a ver cuáles son los ejes tanto retóricos como en cuanto a los tópicos que contienen, basados en la imagen de la mujer ideal del renacimiento, recatada, piadosa y devota de su familia, lo que esperamos contribuya a aclarar la idea, errónea, que de muchas de estas mujeres y de su rol social y político tenemos en la actualidad y que es necesario repensar y reescribir pues todas las mujeres estudiadas tuvieron un papel relevante en la vida política, gobernaron por derecho propio o en ausencia de sus maridos o fueron regentes mientras sus hijos eran menores, sin embargo, su memoria celebra sus virtudes morales cristianas, como hijas, esposas o madres y no su ejercicio del poder.

El estudio de las obras aquí presentadas, y de otras similares, requiere de una mayor pausa, de un corpus mayor y de un análisis más profundo que identifique las tradiciones discursivas, lingüísticas y los motivos que se repiten, pero también aquello que los diferencia, habida cuenta de las características propias y del papel social y político que tuvieron este tipo de textos. 


\section{Referencias bibliográficas}

Allo Manero, María Adelaida \& Esteban Llorente, Juan Francisco. 2004. El estudio de las exequias reales en la Monarquía hispana: Siglos XVI, XVII y XVIII. Artigrama 19: 39-94.

Allo Manero, María Adelaida. 1993. Las exequias reales de la Casa de Austria en España, Italia e Hispanoamérica. Artigrama 10: 597-602.

Amft, Camilla. 2018. Des präteritale Konzept im Frühneuhochdesuschen. Zur Distribution von Präteritalem Perfekt in Flugschriften des 16. Jahrhunderts. Heidelberg: Winter.

Baernstein, P. Renée. 2006. Sposa, figlia, sorella e vecchia matre”. Invecchiare donna in età moderna, tra demografia e cultura. Storia delle donne 2: 212-230.

Bandini, Marco \& Beer, Marina. 1989. Guerre in ottava rima. Guerre d'Italia (1483-1527). Modena: Panini (4 vol.).

Bell, Rudolph M. 1999. How to Do It: Guides to Good Living for Renaissance Italians. Chicago: University of Chicago Press.

Benavent, Júlia. 2011. Las representaciones teatrales ofrecidas a la llegada de Lucrecia a Ferrara. En Romera, Irene \& Josep Lluís Sirera (ed.) La mujer: de los bastidores al proscenio en el teatro del siglo XVI. Valencia: PUV, 27-40.

Benavent, Júlia. 2015. Las relaciones italianas sobre la Conquista de Granada en el siglo XV. En García López, Jorge \& Boadas, Sònia (ed.). Las relaciones de sucesos en los cambios políticos y sociales de la Europa Moderna. Barcelona: Universitat Autònoma de Barcelona, 103-108.

Bertomeu, María José. 2009. Relaciones de sucesos italianas sobre la boda de Felipe II con María Tudor. Cartaphilus 5: 6-17.

Bertomeu, María José. 2014. Aproximación a los textos italianos del siglo XVI entorno al funeral de Carlos V. e-Spania 17: 1-12

Bertomeu, María José. 2018. La mujer invisible: aproximación a los tratados italianos sobre las viudas en el siglo XVI. RSEI 11: 39-50.

Bulgarelli, Tullio. 1967. Gli avvisi a stampa in Roma nel cinquecento. Roma: Instituto di Studi Romani editori.

Chemello, Adriana. 1985. L'Institution delle donne di Lodovico Dolce ossia l'insegnar virtú et honesti costumi alla Donna. En Riondato, Ezio (ed.) Trattati scientifici nel Veneto fra il XV e XVI secolo. Vicenza: Neri Pozza, 103-134.

Ciappelli, Giovanni \& Nider, Valentina (ed.). 2017. La invención de las noticias. Las relaciones de sucesos entre la literatura y la información (siglos XVI-XVIII). Trento: Università degli Studi di Trento. 
Domínguez Casas, Rafael. 2010. Exequias borgoñonas en tiempos de Juana I de Castilla. En Zalama Rodríguez, Miguel Ángel (dir.). Juana I en Tordesillas: su mundo, su entorno. Valladolid: Ayuntamiento de Tordesillas.

Ettinghausen, Henry. 2013. Los avvisi a stampa: las relaciones de sucesos italianas en relación con las españolas. En Andrés, Gabriel (ed.). Protogiornalismo e letteratura. Milano: Franco Angeli, 13-23.

Giachino, Luisella. 2013. Un panegirico per l'Infanta. Le orazioni funebri. In Raviola, Blythe Alice \& Varallo, Franca (ed.) L'Infanta Caterina d'Austria, duchessa di Savoia (1567-1597). Roma: Carocci, 481-498.

Gómez-Salvago Sánchez, Mónica. 1998. Fastos de una boda real en la Sevilla del quinientos (estudio y documentos). Utrera: Grafitrés, S.L.

Infelise, Mario. 2017. Scrivere gli avvisi: autori ignoti e autori di fama. En Ciappelli, Giovanni \& Nider, Valentina (ed.), 19-30.

Ivaldi, Cristina. 1989. Cantari e poemetti bellici in ottava rima: la parabola produttiva di un sottogenere del romanzo cavalleresco. In Hempfer, Klaus (ed). Ritterepik der Renaissance. Akten des deutsch-italienschen Kolloquiums Berlin 1987. Stuttgart: Steiner, 35-46.

Kelso, Ruth. 1978. Doctrine for the lady of the Renaissance. Chicago: University of Illinois Press.

Lopezosa Aparcio, Concepción. 2011. Solemne despedida. Brillante memoria. Las exequias de María Luisa de Orleáns en Palermo a través de la relación de Francismo Montalbo. Pecia Complutense 8(14): 39-53.

Murphy, Caroline P. 2001. Il ciclo della vita femminile. Norme comportamentali e pratiche di vita. In Matthews Grieco, Sara F. \& Brevaglieri, Sabina (ed.) Monaca, moglie, serva, cortigiana: Vita e immagine delle donne tra Rinascimento e Controriforma. Firenze: Morgana edizioni, 15-47.

Pascual, María. 2017. El matrimonio de Felipe II y María Tudor en la correspondencia del Cardenal Granvela. [Tesis Doctoral inédita]. Valencia: Universitat de València, Dept. de Filologia Francesa i Italiana.

Pena Sueiro, Nieves. 2017. Los autores de relaciones de sucesos: primeras precisiones. En Ciappelli, Giovanni \& Nider, Valentina (ed.), 491-508.

Petta, Massimo. 2017. Il racconto degli avvenimenti tra poesia e prosa: la reinvenzione delle notizie da parte degli stampatori. En Ciappelli, Giovanni \& Nider, Valentina (ed.), 543-566.

Pucci, Paolo. 2015. Finalmente libera ma non per molto: La vedova nella trattatistica italiana del XVI secolo. Rivista di studi italiani, anno XXXIII (1): 187-214.

Ricci, Laura. 2009. La lingua degli avvisi a Stampa (secolo XVI). In Cannata, Natalia \& Grignani, Maria Antonietta (ed.) Scrivere il volgere tra Medievo e Rinascimento. Pisa: Pacini editore, 97-114. 
Sanson, Helena. 2015b. Widowhood and Conduct in Late Sixteenth-Century Italy: The Unusual Case of La Vedova del Fusco (1570). The Italianist, 35(1): 1-26.

Solís de los Santos, José. 2007. Peripecia germana de la relación hispalense sobre la boda de Carlos V. In Bolaños, Piedad; Domínguez Guzmán, Aurora \& De los Reyes Peña, Mercedes (coord.) Geh hin und lerne: homenaje al profesor Klaus Wagner. Sevilla: Universidad de Sevilla.

Solís de los Santos, José. 2009. Relaciones de sucesos de Inglaterra en el reinado de Carlos V. En Fernández F. Manuel; González Alberto, Carlos \& Maillard, Natalia (ed.) Testigo del tiempo, memoria del universo. Cultura escrita y sociedad en el mundo ibérico (siglos XV-XVIII). Málaga: Ediciones Rubeo, 640-698.

Varela, Javier. 1990. La muerte del rey: el ceremonial funerario de la monarquía española, 1500-1885. Madrid: Turner.

Wilhelm, Raymund. 1996. Italianische Flugschriften des Cinquecento (15001550). Gattungsgeschichte und Sprachgeschichte. Tübingen: Niemeyer.

Wilhelm, Raymund. 2019. Le donne degli Asburgo in opuscoli e fogli volanti italiani del Cinque e Seicento. Tradizioni discorsive e registri linguistici. Seminario de investigación organizado por el proyecto MAUSTRIA. Valencia: Universitat de València, 10 de abril. 
